\title{
The Nature of Adaptive Interactions and Potential for Strategic Application
}

\author{
Justin Davis \\ Madera Labs, USA \\ justin@maderalabs.com
}

\begin{abstract}
The ability for an interaction with a user to adapt over time can aid in the creation of more engaging user experiences as well as helping to drive business growth through strategic application. These adaptations may take two broad forms: active and passive, with each exhibiting certain characteristics depending on context of use. Each of these models is rooted in the system model for adaptation, a 4-step cycle of information gathering, induction and response that forms the foundation for designing the nature of an adaptive system. In this paper, we describe the nature of adaptation, explore various contexts of use with modern examples and describe potential strategic business application for this interaction methodology.
\end{abstract}

Keywords: adaptive interactions, adaptation, interaction design.

\section{Introduction}

Historically, many designed human computer interactions have involved engagement that is primarily static, starting and stopping with the span of time that the user is actively using the software, and failing to transcend that period in a meaningful and engaging manner. Today, with the increasingly ubiquitous nature of computing, nearly infinite data storage scenarios and an array of new interaction modes via hardware and software combinations, this static nature of software begins to reveal its limitations. As new understanding emerges about how humans interact with technology, and how technology can play an ever-increasingly persistent role in their lives, the role of adaptive interactions becomes not only more feasible, but a key player in the design of more engaging, persuasive and delightful human-computer interactions.

The notion of adaptive interactions - particularly with regards to an interface that changes over time according to user behavior - is not novel. Per Langley:

An adaptive user interface uses traces of its interactions with users to automatically build a model that influences the systems behavior to better suit the user's preferences. (Langley, 1999) 
This alteration of the user interface according to patterns of behavior found in data has been explored in scientific projects like INCA (Gervasio, Iba \& Langley, 1999) and SYSKILL \& WEBERT (Pazzani, Muramatsu \& Billsus, 1996), as well as popular internet websites like Amazon and Netflix. In all of these instances, the ability for an interface to adapt to a user causes the computer to deliver a more relevant and targeted response to the user, thereby increasing efficiency and driving loyalty.

For the purposes of brevity, we will use the word "device" to refer to the item the user is interacting with. This is used with the understanding that this may refer to a computer-enabled device, but also to a human-to-human interaction in the case of adaptation in the service design sector.

\section{Dimensions of Adaptation}

Adaptive interactions require that new dimensions be considered in the design process. Whereas static interactions only require the consideration of the standard three dimensions, designing for adaptation requires the active exploration of at least one additional dimension: time. In addition to time, the dimension of touchpoints might also be considered in more ubiquitous and pervasive applications.

Because adaptation happens by introspecting past performance and altering the state of the object for future performance, the consideration of time is a requirement in the design of adaptive interactions. To design experiences that successful leverage the potential of adaptive interactions, designers must think of time as a primary design constraint and use it to frame the problem space from the outset.

Time is a required component of adaptive interactions, for if there was no record of data over time, there would be no basis on which to craft an adaptation. In addition to time, the dimension of touchpoints (broadly: location) plays an important part in helping the designer to craft engaging adaptive interactions.

From a systemic view, touchpoints represent discreet devices or locations where a user interacts with a device. Salient to this discussion is the requirement that these devices be able to track the user and their behavior, store that data, and use that data across the system for changing the nature of the interaction over time. While not required for the construction of adaptive interactions, the dimensionality of touchpoints does provide the designer with a compelling set of opportunities for extending the interaction past a static object.

\section{A System Model for Adaptation}

Adaptation can be described as a loop with four discreet action points. This loop constitutes the cycle by which data is collected, analyzed, and acted upon for the purpose of changing the nature of the interaction with the user. 


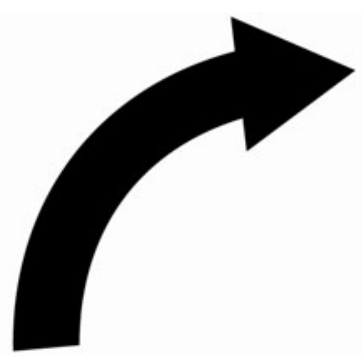

Interaction

Adaptation

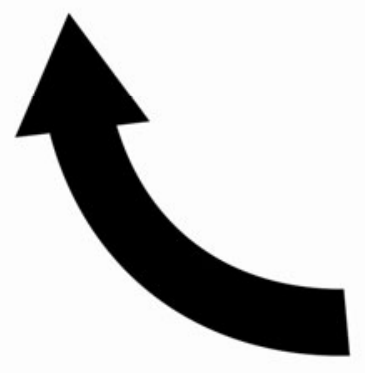

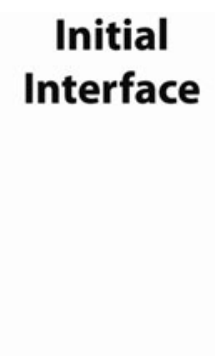

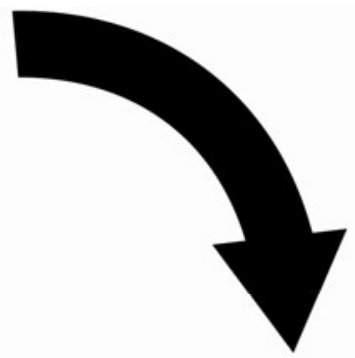

User Response
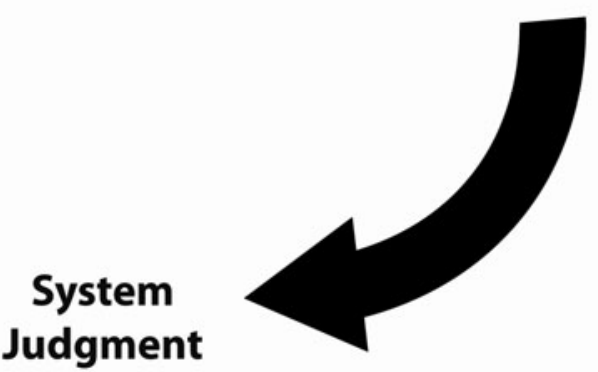

Fig. 1. A System Model for Adaptation

\subsection{Intial Interface}

The initial interface represents the state of the device before the user interacts with it. This happens both at the start of the engagement with the device - when the user is using the device for the first time - as well as at the completion of this loop, after the interaction has adapted and before the user interacts with the device again.

The initial interface can be thought of as being in a "listening" state, passively waiting for the user to act upon it.

\subsection{User Response}

Immediately following the display of the initial interface to the user is the user's response, where an action is applied to the interface. For an interaction to be adaptive, this response must be stored for later retrieval and analysis. Per our earlier discussion regarding time, user responses should be attached to a specific timestamp, so that induction may be carried out with the history of responses intact and chronological.

User response may fall into one of two broad categories: active and passive. In an active response (later discussed as an "Active Adaptation"), the user consciously and intentionally acts upon the interface to drive the adaptation, aware of the adaptive nature of the interaction and their relative effect on it. In a passive response (later discussed as a "Passive Adaptation"), the user does not drive the adaptation proactively. In this model, the system collects behavioral data on behalf of the user by evaluating behaviors over time, as opposed to the use of concrete decision points. 


\subsection{System Judgment}

After a user has taken action on the device, the device uses the data collected from this interaction to derive a conclusion about what the user's behavior means. This is an inductive process, and may be handled by a myriad of predictive modeling and machine learning algorithms. Because the focus of this paper is on the design of adaptive interactions - not the specific mathematical formulation of inductive techniques - we will not cover in detail the specific mathematical formulas needed to carry out this induction. Moreover, Langley and Simon (1995) note that the particular induction method is less important than the steps of problem formulation, representation engineering and data collection/preparation.

Salient to this discussion is that the system must use user behavior and/or response data to formulate the basis for adapting the interaction before the next session with the user.

\subsection{Interaction Adaptation}

During the final phase of this system model, the device must actually execute the necessary alterations to the interaction before the user interacts with the device again, thus starting the loop over. These alterations may be tangible to the user, such as a change to the user interface or information being served to the user, or may be intangible, such as the adjustment of the user model internally within the system. It should be noted that the point of adaptive interactions is to eventually change the tangible interaction with the user, so although a device may spend some cycles of this loop adjusting the user model instead of the interface, the tangible adjustment of the interaction is eventual.

\subsection{Two Models of Adaptation: Active and Passive}

Two main implementation methodologies exist for adaptive interactions: active and passive. Each of these describes the nature of the user's role in driving the adaptation, and has consequence on the algorithms selected to drive the change, as well as the design of the interface itself.

\subsection{Active Adaptation}

In an actively adaptive model, the nature of the interaction changes as a result of a conscious and intentional effort on the part of the user to drive this adaptation. These systems actively solicit input from the user, using the user's response to help drive the inductive process for the adaptation.

Actively adaptive interactions display certain characteristics that help to define the model. With an actively adaptive model, the user interface must offer the user decision points to drive the adaptation. These decision points generally take a binary, yes/no form, allowing the user to play a persistent role in crafting the adaptation. These decision points may offer explicit choices that map directly to the construction of the algorithm, or may offer choices that are more implicit in nature. These implicit choices utilize associated metadata to help drive the inductive process. However, regardless of the explicit or implicit nature of the user's input, actively adaptive systems rely on a user driving the nature of the adaptation directly through decision points. 
One advantage of actively adaptive systems is the simplicity by which the predictive algorithms may be implemented. Because the system is changing behavior due to a user's overt and explicit instruction, the reliance on predictive modeling algorithms is lessened. From an implementation standpoint, these models "learn" by offering the users choices, observing the choice and making the same choice later for the user. They may also map the metadata from a user's implicit choice to other possible outputs, adjusting the information served to the user according to profiles attached to these decision points. Although more simple to implement, actively adaptive systems require a greater degree of involvement on the part of the user, and may be less compelling than passively adaptive interactions in terms of experience.

Pandora, based on the work of the Music Genome Project, is an example of an actively adaptive interaction. The application works by soliciting input from the user as to what type of music they would like to listen to. This input may be in the form of artist, song or genre. After the user has supplied this data to the application, Pandora proceeds to play music that is similar, based on metadata attached to each piece of media.

While listening, a user is able to adjust the accuracy of the station by giving a "thumbs up" or "thumbs down" to individual songs. This action is the required active input for the active adaptation. After indicating their preference, Pandora adjusts its algorithm by examining the characteristics of that song, and serving up future music based on the user's preferences. This is an implicit decision point, where the user is instructing to the system that they do or do not like a certain song, and using the associated metadata for that song to drive the adjustment of the inductive process. Pandora also implements an explicit decision component in this process, whereby a particular song is no longer played after two "thumbs down" by a user. The difference in implicit and explicit decision points is quite clear here: with an implicit decision point, the user's feedback is extrapolated to other instances using attached metadata, whereas an explicit decision point instructs the system as to how to behave in the exact circumstance in the future (in this case, not playing the exact song that the user has applied the feedback to).

\subsection{Passive Adaptation}

In a passively adaptive model, the nature of the interaction changes automatically over time, through induction of the user's past behaviors. These systems do not require active solicitation of the user, instead adjusting the interaction automatically in subtle but meaningful ways over time.

Because of the heavy reliance on induction, passively adaptive systems are more difficult to implement, and require more careful examination of the data collection and representation methods used, to assure that the alterations in the model are representative of a user's actual desires.

Passive adaptation also carries with it extra implementation burden not always associated with actively adaptive models. In a passively adaptive model, the potential for confusion and disorientation on the part of the user is greatly increased, due to the fact that the user is not inherently involved in the adaptation of the interaction. As a system adapts to better serve a user, the nature of these adaptations must be smaller and more incremental than in that of an actively adaptive system. Said another way, 
much more behavioral data is needed to effect changes in a passively adaptive interaction than is needed in an actively adaptive model.

In addition to the potential for confusion and disorientation, passively adaptive interactions must also employ override mechanisms for allowing users to adjust the adaptation manually, or correct an errant assumption made by the algorithm. These overrides act as an adjunctive actively adaptive component, and demonstrate that passively adaptive interactions may not perform well as purely passive, but as a blend between the two modes.

Passively adaptive interactions are becoming more common, especially in the web domain. Google's recent product, Priority Inbox, is an example of such a system, and employs the aforementioned characteristics of a passively adaptive system.

With Priority Inbox, the system attempts to use induction of user behavior to establish priority in an email inbox. It does this by using inductive methods, observing user behavior such as who you email, which messages you open, which keywords appear in messages commonly opened and which messages you reply to (Google). However, as is characteristic of a passively adaptive interaction, the system also features override mechanisms, in the form of buttons that allow the user to manually label a message important or not important. Per our earlier discussion, these override mechanisms are strongly encouraged in a passively adaptive interface to allow the user to adjust the nature of the adaptation, should induction prove inaccurate.

\subsection{Adaptive Interactions as a Strategic Business Tool}

The use of adaptive interactions can be leveraged as a significant driver of business in a transactional situation. Adaptive interactions have the ability to deliver value to a user in more poignant and relevant ways, which can drive revenue through an increase in sales conversion and by intentionally raising the switching costs associated with leaving a provider.

Amazon has demonstrated the efficacy of adaptive interactions as a strategic business tool. By using product recommendations - using adaptive models such as content-based and collaborative filtering (Langley, 1999) - Amazon has increased revenue by increasing the likelihood that that users will convert, due to an increased relevancy of the information being displayed to them. These recommendations have accounted for up to 35\% of Amazon's yearly revenue (VentureBeat), demonstrating the power of adaptive interactions for driving revenue-generating conversions. The adaptation models used by Amazon to display recommendations are of the passive class, learning from user behavior over time to display more relevant content in future interactions.

In addition to increasing conversion, adaptive interactions can also drive revenue creation by intentionally raising the switching costs associated with leaving a provider. Burnham, Frels and Mahajan (2003) describe personal relationship loss costs as losses that may be incurred when a bond is broken between a customer and the people with whom that customer interacts. This relationship may be bolstered or defined by a rich data set and existing history shared between customer and provider, which allows the provider to deliver a more relevant experience through the use of adaptive interaction. Should the customer leave a provider with which they have a long history, they lose the benefit and comfort associated with the former provider. 
This increased switching cost speaks to the benefit of leveraging user data to create more relevant experiences, exposing the potential switching costs to the user and driving deeper loyalty with the provider.

\section{Concluding Thoughts}

Clearly, the ability for an interaction to adapt to a user carries with it both compelling experience advantages as well as business growth opportunities. The nature of adaptation is complex, consisting of varying interaction methodologies, inductive algorithms and strategic business considerations. Despite this complexity, the potential of adaptive systems to deliver a more engaging and relevant experience to users is exciting. To be successful, a designer must possess an understanding the user's experience over the dimensions of time and touchpoint, and understand how to design each discreet moment throughout the system model for adaptation. In the application of adaptive interactions, the devil is in the details; care must be taken to carefully consider the correct adaptive scenario to be used, and each component of the adaptive interaction must be designed with caution and intent. Executed successful, however, adaptive interactions can create exciting new moments for people interacting with technology and can aggressively drive business campaigns.

\section{References}

1. VentureBe, http: / /venturebeat.com/2006/12/10/ aggregate-knowledge-raises-5m-from-kleiner-on-a-roll/

2. Burnham, T., Frels, J., Mahajan, V.: Consumer Switching Costs: A Typology, Antecedents, and Consequences. Journal Academy of Marketing Science 31(2), 109 (2003)

3. Gervasio, M.T., Iba, W., Langley, P.: Learning User Evaluation Functions for Adaptive Scheduling Assistance. In: Proceedings of the Sixteenth International Conference on Machine Learning, Bled, Solvenia, Morgan Kaufmann, San Francisco (1999)

4. Google,

http://mail.google.com/support/bin/

answer. py?hl=en\&answer $=186543$

5. Langley, P.: Machine Learning for Adaptive User Interfaces (1997)

6. Langley, P.: User Modeling in Adaptive Interfaces. In: Proceedings of the Seventh International Conference on User Modeling, Banff, Alberta. Springer, Heidelberg (1999)

7. Pazzani, M., Muramatsu, J., Billsus, D.: SYSKILL \& WEBERT: Identifying interesting web sites. In: Proceedings of the Thirteenth National Conference of the American Association for Artificial Intelligence, pp. 54-61. AAAI Press, Portland (1996) 